\section{PSICOLOGÍA IBEROAMERICANA}

\section{Psicología lberoamericana}

ISSN: 1405-0943

psicología.iberoamericana@uia.mx

Universidad Iberoamericana, Ciudad de

México

México

Acevedo Ponce de León, Juan Antonio; Carrillo Árcega, María de Lourdes Adaptación, Ansiedad y Autoestima en Niños de 9 a 12 años: una Comparación entre Escuela

Tradicional y Montessori

Psicología Iberoamericana, vol. 18, núm. 1, enero-junio, 2010, pp. 19-29

Universidad Iberoamericana, Ciudad de México

Distrito Federal, México

Disponible en: http://www.redalyc.org/articulo.oa?id=133915936003

- Cómo citar el artículo

- Número completo

- Más información del artículo

Página de la revista en redalyc.org

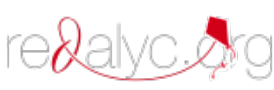

Sistema de Información Científica

Red de Revistas Científicas de América Latina, el Caribe, España y Portugal

Proyecto académico sin fines de lucro, desarrollado bajo la iniciativa de acceso abierto 


\title{
Adaptación, Ansiedad y Autoestima en Niños de 9 a 12 años: una Comparación entre Escuela Tradicional y Montessori
}

\author{
Adjustment, Anxiety and Self-Esteem in Students Between 9 and 12 years old: \\ A Comparison Between Traditional School System and Montessori System
}

\author{
Juan Antonio Acevedo Ponce de León \\ María de Lourdes Carrillo Árcega * \\ Universidad de las Américas Puebla, México
}

\section{RESUMEN}

La adaptación, sea biológica o psicológica, es un proceso fundamental que, cumplido cabalmente, propicia el desenvolvimiento armónico del individuo en el ambiente y en la sociedad, garantizando incluso su sobrevivencia. Dada la consideración que diversos autores hacen tanto de la ansiedad como de la autoestima en tanto mecanismos adaptativos, se hace pertinente explorar la correlación existente entre la adaptación y estas dos variables. Adicionalmente, se indaga si las características y prácticas específicas de dos diferentes métodos educativos señalan diferencias claras en los índices de adaptación, ansiedad y autoestima entre sus alumnos. Por tanto, en el presente trabajo se describen y comparan los niveles de adaptación, ansiedad y autoestima registrados en 72 alumnos de escuela tradicional y 65 de escuela Montessori de la ciudad de Puebla, de entre 9 y 12 años de edad.

Se describen, además, resultados del análisis factorial aplicado a los tres instrumentos psicométricos utilizados en esta investigación, dos de los cuales fueron generados en España y otro en Estados Unidos de América.

Descriptores: Adaptación, ansiedad, autoestima, niñez, estructura factorial, método tradicional, método Montessori.

\section{ABSTRACT}

The adaptation, be it biological or psychological, is a fundamental process that, when it is fully met, fosters the harmonious development of the individual within the environment and the society, including ensuring its survival. Given that several authors consider both the anxiety and self-esteem as adaptive mechanisms, it becomes relevant to explore the correlation between adaptation and these two variables. Additionally, it explores whether specific characteristics and practices of two different educational methods determine differences in the rates of adjustment, anxiety and self-esteem among their students. Therefore, this paper describes and compares the levels of adaptation, anxiety and self-esteem recorded in 72 students from traditional school and 65 from Montessori school in the city of Puebla, between 9 and 12 years of age.

We describe further results of factor analysis applied to the three psychometric instruments used in this investigation, two of which were generated in Spain and another one in the USA.

Key words: Adjustment, self-esteem, anxiety, childhood, factorial structure, traditional school system and Montessori system.

* Juan Antonio Acevedo Ponce de León. Universidad de las Américas Puebla. No. telefónico: (Lada 01/222) 22920 00, ext. 2055. Correo electrónico: juann.acevedo@udlap.mx • María de Lourdes Carrillo Árcega. Universidad de las Américas Puebla. No. telefónico: (Lada 01/222) 233 53 16. Correo electrónico: lourdes.carrillo@gmail.com. 


\section{INTRODUCCIÓN}

La escuela es, además de la familia, el contexto fundamental que provee al niño de las condiciones y elementos para su desarrollo físico y psicológico, así como para su interacción con el medio ambiente. Para alcanzar tal propósito, cada método educativo considera como elementos del proceso educativo a los alumnos, profesores, así como al contenido, contexto institucional y tecnología educativa. La diferencia entre las distintas propuestas pedagógicas, como ocurre en el caso concreto del método tradicional y del método Montessori, estriba en que cada uno acciona dichos componentes desde perspectivas diametralmente opuestas. Por ejemplo, el sistema Montessori se centra en las necesidades del niño, utiliza una gran variedad de materiales sensoriales; cada niño toma su tiempo para aprender; al interior del aula, el menor elige un lugar para cumplir con su trabajo; en un mismo espacio aprenden juntos alumnos de distintas edades. En contraparte, en el sistema tradicional priva el magistrocentrismo, hay escaso uso de materiales sensoriales, el alumno dispone de tiempo limitado para cumplir sus tareas, tiene asignado un lugar fijo en el salón, los grupos generalmente están formados por niños de la misma edad. El análisis de estas prácticas implica, obviamente, la consideración de estructuras y procesos más amplios y complejos.

Surge entonces la interrogante sobre si los procedimientos y estrategias propios de cada sistema, propician diferencias en niveles de adaptación, ansiedad y autoestima entre los alumnos de una y otra modalidad educativa; adicionalmente se examina la correlación existente entre las tres variables mencionadas.

La psicología, a través de la corriente funcionalista, retoma el concepto de adaptación, entendiéndolo como ajuste al medio a través de la promoción de cambios conductuales. Este término fue aportado por Darwin (1859/2005) quien, desde la perspectiva biológica, se refirió a su importancia: "La más pequeña ventaja en ciertos individuos, en cualquier edad o estación, sobre aquellos con quienes entran en competencia, o la mejor adaptación, por pequeño que sea el grado, a las condiciones físicas ambientes, harán a la larga inclinar la balanza a su favor”. (p. 455)

Partiendo de la concepción del hombre como "fenómeno” biológico, psicológico y social, Merani (1978) explicaba que mientras que los animales dependen de su aparato biológico para la adaptación, los seres humanos cumplen un proceso doble de adaptación: por un lado el "comunitario", similar al de los animales, que consiste básicamente en adaptarse al grupo y por otro lado el "individual" que le es exclusivo y que le permite acceder a un nivel superior donde le es dado prever y sintetizar. La trascendencia del fenómeno de adaptación reside en que, tanto en sentido biológico como psicológico, garantiza la sobrevivencia de los seres humanos.

Por su parte, Schaffer (2000) ha considerado rebasados los cuestionamientos sobre si el niño se "hace" o "nace" social, pues en su opinión el niño nace "preadaptado" a la vida social, lo cual es una capacidad análoga a su disposición a admitir alimento y respirar.

La adaptación ha sido abordada desde distintas perspectivas, por ejemplo, López y Cantero (2004) examinaron la adaptación escolar en niños de 3 años de edad, a través de su estado emocional y conductas emitidas tanto en el hogar como en la escuela. Los datos analizados fueron facilitados por los propios niños, sus padres y maestros. Los investigadores no encontraron diferencias significativas en la adaptación escolar de la mayoría de los sujetos, aunque un 10\% de ellos no alcanzaron niveles aceptables en esta variable. En su opinión, estos resultados sugieren revisar la concepción generalizada acerca de la adaptación escolar como un proceso "traumático y universal".

Por su parte, Carreras, Muñoz, Braza Lloret y Braza (2002) estudiaron el probable valor predictivo de la "flexibilidad comportamental" en la adaptación social posterior. Para tal propósito aplicaron, en un primer momento, a niños entre 4 y 5 años de edad, un instrumento generado por este equipo de investigadores denominado Índice de Flexibilidad Comportamental (IF); cuatro años más tarde, administraron a la muestra original el Test Autoevaluativo Multifactorial de Adaptación Infantil (TAMAI). Los resultados obtenidos, así como las correlaciones identificadas entre algunos factores familiares del TAMAI y el IF, permiten corroborar el valor predictivo de este último sobre la ulterior adaptación social.

En cuanto a la adaptación psicosocial positiva (resiliencia), Pesce (2004) investigó la relación que tiene esta variable con experiencias de vida adversas y "factores de protección" en una muestra conformada por 
adolescentes. Si bien no estableció correlación entre resiliencia y situaciones difíciles, sí la halló entre resiliencia y "factores de protección": familia, amigos y profesores.

Sobre la adaptación afectiva y social de niños entre 9 y 14 años, Gallardo y Jiménez (1997), abordaron las consecuencias que tiene el "maltrato y rechazo sociométrico" sobre esta variable. Concluyeron que recibir maltrato físico o presenciarlo provoca niveles similares de desadaptación y que sujetos que se consideran "rechazados" presentan un mayor índice desadaptativo.

Respecto a la ansiedad, Echeburúa (1998) ha establecido que esta consiste en una reacción esperada, propia de la adaptación, ante peligros concretos o productos de la fantasía, vagos o confusos, que alertan al organismo para responder ante el riesgo. El autor afirma que cuando la ansiedad surge de estímulos determinados, se le denomina miedo y resulta común que los menores perciban diversos temores cuya intensidad, duración y tipo difieren según la edad y se inactivan con el tiempo.

Por su parte, Agras (1989) catalogó al miedo y al dolor en dos niveles diferentes; para él el miedo es un mecanismo primigenio de alerta que propicia que el niño se sustraiga de riesgos, en la medida en que el dolor le previene de un perjuicio físico inmediato. En cuanto a los miedos, en primera instancia enlistó el "miedo a la separación" y después de éste a "los miedos a los animales y los daños físicos". Definió a los miedos como reacciones universales no aprendidas e instintivas, cuya finalidad sería la de preservar a los infantes de riesgos diversos.

Gray (1971) segmentó los "miedos innatos" en cinco categorías: miedo a los "estímulos intensos" (dolor, sonidos), miedo a los "estímulos desconocidos" (gente extraña), miedo a la "ausencia de estímulos" (apagar la luz), miedo a "estímulos potencialmente peligrosos" identificados filogenéticamente (grandes altitudes, separación, animales ponzoñosos) y miedo a "interacciones sociales con desconocidos". No obstante, Echeburúa (1998) advirtió que investigaciones en torno a estas afirmaciones han puesto en duda, en cierta medida, tanto su naturaleza instintiva como su universalidad.

En lo que concierne a la ansiedad en el niño y en el adolescente, Castro (1998) realizó una exhaustiva revisión documental que le permitió establecer que si bien la ansiedad infantil emerge como un fenómeno heterogéneo, sus expresiones son distintas para cada edad y contexto en que el niño se desarrolla.

Sobre la ansiedad en la niñez, González y García (1995) han afirmado que son múltiples los factores que pueden producir "desequilibrio" entre las demandas de los estímulos y las respuestas del niño: nacimiento de hermanos, ingreso a la escuela, exigencias familiares, divorcio o separación de los padres; eventos que provocan que el infante pueda sentirse desbordado, inadaptado.

A la autoestima, Branden (2001) la ha descrito como el resultado de la evaluación que las personas elaboran sobre sí mismas y las impacta en el aspecto psicológico y motivacional. El individuo percibe tal valoración como una emoción, como un sentimiento asociado a otros sentimientos, por lo que resulta complejo reconocerlo, separarlo; no le es posible apreciarlo conscientemente. La emoción es considerada por este autor como resultado de una estimación de la interacción entre el yo y un elemento de la realidad.

A su vez, Pruneda (2003) reconoce a la autoestima como un fenómeno complejo, dado que es construida por cada ser humano a partir de las aportaciones que realizan figuras significativas con que se relaciona desde la infancia, de las que aprende a sentirse bien o mal consigo mismo; de esta manera se valora a sí mismo. La autoestima es un proceso individual, que en la actualidad se dificulta por transformaciones sociales, económicas y políticas que provocan un incremento en el estrés que el sujeto experimenta ante las demandas para ser mejor, más productivo, más eficiente.

Hess y Croft (1981) coincidieron con lo anterior al considerar a la autoestima como factor fundamental de la "triple conquista" del niño, conjuntamente con el autoconcepto y la identidad sexual. En opinión de estos autores, la autoestima representa el grado de valoración que el sujeto confiere a lo que hace, a lo que él mismo es y lo que siente al respecto. Explican que en el proceso de autoestima el individuo incorpora gradualmente apreciaciones negativas y positivas de sí mismo, que proceden de la retroalimentación con figuras significativas para él, como sus padres y maestros. Montt y Ulloa (1996) asentaron el papel determinante de la autoestima en aspectos tan esenciales como el rendimiento escolar, los grados de ansiedad, 
la adaptación a nuevas circunstancias, las relaciones interpersonales y la aceptación de otros individuos. En una muestra constituida por adolescentes, las autoras lograron establecer la relación entre baja autoestima y trastornos psicológicos.

Verduzco, Lucio y Durán (2004) exploraron la influencia de la autoestima en la identificación del estrés y el estilo de afrontamiento utilizado por 339 alumnos de cuarto, quinto y sexto de primaria. Descubrieron que la recurrencia a tipos de afrontamiento indirecto o de abandono de control se incrementa en sujetos con menor autoestima, tendencia que se invierte cuanto más elevada resulta esta variable.

Palacios, Marchesi y Coll (2002) han establecido que las dimensiones sustanciales de la autoestima van variando con la edad, tanto en niños como en niñas, tendencia que está determinada por el propio desarrollo evolutivo, donde procesos como la autoestima se presentan cada vez más complicados y diversificados.

Bordera (2005) ha hecho referencia a la posibilidad de que la escuela se constituya en instancia de apoyo a estudiantes que experimenten episodios traumáticos como: terrorismo, muerte de seres queridos, accidentes, violencia y acoso en la escuela (bulling). Tales experiencias, en mayor o menor medida, suelen conllevar consecuencias negativas y su solución generalmente se supedita al ámbito clínico o familiar. Su propuesta reside en proporcionar a los docentes las herramientas para intervención una oportuna y eficaz en estos casos.

Emprender el análisis de la educación resulta una tarea compleja, dado que deben considerarse no sólo características y procesos internos como la enseñanza, el aprendizaje, el clima escolar, la comunidad educativa y el estilo docente, entre otros. Debe ser incorporado además, el estudio de factores externos que determinan el proceso educativo desde su origen hasta su estado actual: contextos políticos, sociales y económicos, situación geográfica, cultura y, en algunos casos, hasta la religión. En respuesta a las demandas de determinado momento histórico, han surgido diversas propuestas pedagógicas que parecen obedecer a un objetivo común: educar. La diferencia entre una y otra, estriba en la concepción que cada corriente tiene del proceso educativo, de sus propósitos explícitos e implícitos, de los procedimientos que elige para cumplirlo y del papel asignado en este proyecto a alumnos y maestros.
Teóricos diversos han hecho aportaciones respecto a múltiples temas educativos. En referencia a la educación tradicional, Di Giorgi (1977) enfatizó la necesidad de subsanar las carencias y las insuficiencias de este tipo de escuela que, según su opinión, residen en su calidad y la convierten en una "institución desadaptante”. De la Garza (2002) al examinar la función docente, encontró que en el método tradicional el proceso educativo gravita alrededor de la enseñanza, la responsabilidad recae exclusivamente en el profesor, quien es considerado guardián del conocimiento. Al interior de este esquema, su acción docente es intuitiva.

Como alternativa al sistema tradicional, Di Giorgi (1977) propuso que la escuela modificara condiciones económicas y sociales a través del cumplimiento de programas que posibiliten que el alumno adquiera conocimientos vinculados a su realidad, en un proceso en el que no sea juzgado y presionado por sus maestros, sino ayudado por éstos. Por tanto, sugirió una "enseñanza activa", estrechamente vinculada a la vida cotidiana, en la que el maestro asuma una actitud activa ante niños activos, en la que la experiencia del niño sea un factor esencial. De la Garza (2002), al hacer referencia al modelo "científico", encontró que los antecedentes teóricos de éste se ubican en las escuelas nueva y activa, su eje lo constituye el aprendizaje y el maestro se asume como estimulador del desarrollo de "habilidades del pensamiento", como guía de sus alumnos para "aprender a aprender". El citado autor anotó también que la escuela "crítica" se manifiesta a través de dos perspectivas paralelas que convergen en esta visión: la "institucional" que se refiere a las implicaciones sociales de la educación y sus instituciones y, por otro lado, la "humanista" que enfatiza sobre la influencia de la educación en la construcción de la "persona humana" en el alumno y en el maestro. En este enfoque el profesor promueve en el educando la "conciencia crítica" sobre la realidad que lo circunda.

Tanto los profesores como los alumnos encuentran la orientación a su quehacer en los principios metodológicos de la propuesta pedagógica a la que se encuentran suscritos. Esta diferencia de estrategias y procedimientos, ¿propicia diferencias estadísticamente significativas en análisis comparativos entre alumnos de métodos tradicional y Montessori? Diversos autores han realizado investigaciones en este rubro sobre 
diferentes variables. Por ejemplo, en un estudio comparativo elaborado por Tirado y Sánchez (1993) sobre las "habilidades de reflexión" en 1084 niños de 11 a 12 años inscritos en escuelas tradicionales y activas o Montessori, hallaron que los alumnos en quienes la escuela fomenta la reflexión (como en el caso de las escuelas Montessori o activas) desarrollan más estas habilidades que los estudiantes de escuelas tradicionales. Otro análisis comparativo entre dos diferentes métodos educativos, se debe a Mishra (1988) quien investigó las diferencias de estrategias verbales de aprendizaje entre alumnos (entre 5 y 11 años de edad) de escuelas tradicional y "moderna". No encontró diferencias significativas en cuanto a estrategias verbales de aprendizaje entre ambas muestras. Sin embargo, identificó diferencias entre los patrones de organización verbal entre las dos escuelas. Por su parte, Jaik, Serrano, López y Amancio (2008) compararon cinco variables asociadas a "áreas de la vida práctica, sensorial, lenguaje, matemáticas y áreas culturales”, en 60 niños inscritos en escuela tradicional o escuela Montessori. Hallaron que los alumnos de método Montessori registraron en las variables estudiadas porcentajes superiores a los alcanzados por niños del modelo tradicional.

En tanto, Gutiérrez, Lerma y Schroeder (2009) investigaron la socialización en 116 alumnos de 11 a 13 años de edad, de los cuales 63 pertenecían a escuela tradicional y 53 a escuela con método Montessori. Aplicando la Batería de Socialización (BAS-3), identificaron mayores puntuaciones en las áreas de "consideración por los demás, autocontrol en las relaciones sociales y sinceridad" en alumnos de escuelas Montessori, lo que llevó a distinguir diferencias significativas en estos rubros. Sin embargo, los investigadores comprobaron que no existían diferencias relevantes en los aspectos "liderazgo, ansiedad social y timidez" así como en "retraimiento social" entre los dos grupos.

En cuanto a la comparación de niveles de autoestima, auto-eficacia, conducta prosocial y comportamiento agresivo entre alumnos de segundo a sexto grado procedentes de escuela tradicional y Montessori, Castellanos y Albizu (2003) no hallaron diferencias significativas en los puntajes correspondientes a autoestima, auto-eficacia en el logro académico y en el aprendizaje autorregulado, así como auto-eficacia social y conducta prosocial. No obstante, se registró una diferencia significativa en la categoría de agresión física o verbal, dado que los alumnos de escuela Montessori presentaron niveles más bajos que los estudiantes de escuela tradicional; condición que permite, en opinión de los autores, que los niños pertenecientes a escuela Montessori desarrollen una habilidad superior a la de los alumnos de escuela tradicional para trabajar al interior de un grupo de iguales, así como para establecer y mantener amistades de su mismo sexo. La capacidad de los alumnos de escuela Montessori para trabajar en equipo se vincula positivamente a altos puntajes en auto-eficacia para logros académicos y en autorregulación del aprendizaje. Así mismo, los autores encontraron que el grado de autoestima de los niños inscritos en el método Montessori, está correlacionado significativamente con sus niveles de auto-eficacia para el logro académico y el aprendizaje autorregulado. Respecto a los alumnos de escuela tradicional, estos mostraron que a temprana edad la auto-eficacia para autorregular el aprendizaje y trabajar en grupo alcanza niveles considerables, sin embargo, con el transcurso del tiempo esta característica decrece.

La presente investigación tiene el objetivo de analizar si las estrategias didácticas, estilos docentes y fundamentos filosóficos propios de dos métodos educativos distintos propician diferencias significativas en los niveles de adaptación, autoestima y ansiedad de los educandos.

Al considerar en conjunto a la adaptación psicológica y la biológica como proceso fundamental en la evolución humana, se analiza el vínculo que esta tiene con la ansiedad, cuya presencia constante en la existencia cumple la función de sustraer al ser humano de riesgos, de preservarlo, lo cual hace factible apreciarla como mecanismo adaptativo. Asimismo se explora la correlación de las dos variables mencionadas con la autoestima, por conceptuarla también como mecanismo adaptativo, dado su papel fundamental tanto en el ajuste a circunstancias nuevas, como en la aceptación del niño por otros sujetos e incluso en la determinación de niveles de ansiedad.

Como prueba de "validez de constructo", se realizó el análisis factorial respectivo de los tres instrumentos psicométricos seleccionados para explorar las variables propuestas en el presente estudio e identificar sus factores, definidos por Coolican (2005, p. 126) 
como "variables ocultas o interventoras" que propician la comprensión de "las relaciones" existentes entre los puntajes obtenidos por los sujetos en distintas "pruebas o subpruebas".

$\mathrm{Al}$ reconocer a la escuela como contexto determinante en la formación de los niños, se pretende analizar si las diferencias en el ambiente, estilo docente, tecnología educativa y perspectiva metodológica entre escuelas tradicionales y Montessori, pudieran propiciar ciertas variaciones en los niveles de adaptación, ansiedad y autoestima de sus alumnos.

Por tanto, el problema de investigación se plantea así: ¿El método educativo (tradicional o Montessori) determina diferencias en niveles de adaptación, de ansiedad y autoestima en niños y niñas (entre 9 y 12 años)?

En concordancia con lo anteriormente expuesto, se propone la hipótesis de investigación: Los niveles de adaptación y autoestima de alumnos entre 9 y 12 años de edad de escuela Montessori son mayores a los de alumnos de las mismas edades de escuela tradicional. Así mismo, los índices de ansiedad en alumnos de escuela Montessori son menores a los de alumnos de escuela tradicional para un rango de edades entre 9 y 12 años.

\section{MÉTODO}

Se planteó un estudio cuantitativo, con alcance descriptivo comparativo, con un diseño no experimental, transeccional y para el cual se realizó un muestreo no probabilístico y propositivo.

Para tal fin, se recurre a grupos "intactos" que cumplen los criterios de edad, sexo, nivel socioeconómico y pertenencia a un grupo escolar (tradicional o Montessori) establecidos para esta investigación. Los puntajes obtenidos por los sujetos en los tres instrumentos aplicados, permiten describir los índices de adaptación, ansiedad y autoestima de la muestra, así como establecer comparaciones entre los niveles alcanzados por alumnos de escuela tradicional y de escuela Montessori en las tres variables estudiadas.

\section{Participantes}

Participaron 137 sujetos de 9 a 12 años de edad, todos ellos alumnos de escuelas primarias, de los que 72 (37 niños y 35 niñas) asistían a escuelas tradicionales y 65 (31 niños y 34 niñas) a escuelas Montessori. Es decir, 68 pertenecían al sexo masculino y 69 al femenino, con una media de edad de 10.14 años. Los criterios de exclusión fueron el de excluir de la muestra a alumnos con necesidades educativas especiales asociadas a discapacidad y eliminar respuestas de niños que después de su aceptación inicial se retiraron de la investigación o bien incurrieron en errores y omisiones al responder los tests.

\section{Instrumentos}

Test autoevaluativo multifactorial de adaptación infantil ([Tamai], Hernández Hernández, 2004).

Descripción: Orientado a la apreciación del grado de inadaptación en menores de 8-18 años, integrado por 175 reactivos, permite identificar al interior de esta variable unos subfactores que, al asociarse, integran clusters o conglomerados que posibilitan establecer las fuentes de la inadaptación. Aborda también las "actitudes educadoras de los padres". Su aplicación fue colectiva. A través del análisis factorial se determinaron 4 factores, la prueba de confiabilidad alcanzó un alfa de Cronbach de .984

Cuestionario para la evaluación de autoestima en educación primaria ([A-EP], Ramos Álvarez, Jiménez López, Muñoz-Addell \& Lapaz Cruz, 2006).

Descripción: El A-EP está diseñado de manera simple, es una sencilla y atractiva prueba para valorar la autoestima en alumnos de cuarto a sexto de primaria. Utiliza un lenguaje accesible adaptado a niños de 9 a 13 años, utiliza viñetas de colores para ilustrar cada uno de los 17 reactivos que lo componen, lo cual facilita el proceso de aplicación (individual o colectiva) y calificación de la prueba. Mediante análisis factorial se obtuvieron 3 factores; la confiabilidad alcanzada fue de .745 de alfa de Cronbach.

Escala de Ansiedad Manifiesta en Niños ([CMAS-R], Reynolds \& Richmond, 1985).

Descripción: La CMAS-R subtitulada "lo que pienso y siento", es un test considerado de "autoinforme", constituido por 37 reactivos, proyectado para evaluar el grado e índole de la ansiedad en niños y adolescentes de 6 a 19 años de edad. Su aplicación puede ser individual o colectiva. En el análisis factorial se identificaron 4 factores, en cuanto a la confiabilidad el alfa de Cronbach resultó de .840 


\section{Procedimiento}

Se presentó a las instituciones seleccionadas, tanto tradicionales como Montessori, el proyecto de investigación con el fin de que aprobaran la aplicación de los instrumentos psicométricos seleccionados a sus alumnos entre 9 y 12 años de edad. Se recurrió a escuelas primarias privadas, bajo la consideración de que las escuelas Montessori son particulares también, y la satisfacción de dicho requisito garantizaría condiciones socioeconómicas análogas entre los sujetos. Las consideraciones éticas que se observaron fueron: información pormenorizada a las escuelas sobre los propósitos de la investigación, naturaleza y contenido de los tests a aplicarse con el fin de obtener su consentimiento. A los niños se les explicó que su participación era voluntaria y que la información que proporcionaran sería tratada en forma confidencial.

A los alumnos de las escuelas que aceptaron colaborar les fue aplicada colectivamente una batería constituida por los tres instrumentos psicométricos descritos en el apartado anterior. Una vez respondidos los tests fueron devueltos a los investigadores para su procesamiento estadístico.

En todos los casos, el escenario determinado para esta fase del proyecto fue el salón de clases, donde una o dos maestras estuvieron presentes durante el desarrollo de las pruebas.

El tratamiento estadístico se realizó utilizando el SPSS versión 13; para contrastar los puntajes de los grupos tradicional y Montessori en las variables adaptación, autoestima y ansiedad se utilizaron pruebas $t$ de Student para muestras independientes; para el análisis factorial se recurrió al método de componentes principales con rotación Varimax.

\section{RESULTADOS}

En cuanto a sexo de la muestra, 68 sujetos pertenecen al sexo masculino y 69 al femenino; de los cuales 72 niños pertenecen a escuelas tradicionales y 65 a escuelas Montessori.
Respecto a la contrastación de la muestra de escuela tradicional y de escuela Montessori, habiendo aplicado la prueba t de Student para muestras independientes, con un nivel alfa de .05, se obtuvieron los siguientes índices (tabla 1).

Valores que permiten corroborar que aún y cuando existen diferencias en las medias registradas en las tres variables, éstas no resultan estadísticamente significativas. Por lo que se afirma que no existen diferencias en los niveles de inadaptación, ansiedad y autoestima entre alumnos de escuela tradicional y Montessori.

La media de edad de la muestra fue de 10.14 años, siendo la frecuencia de edad como sigue: treinta y tres niños de 9 años, sesenta y tres de 10 años, treinta y uno de 11 años y once de 12 años.

En cuanto al análisis de las correlaciones existentes entre inadaptación, ansiedad y autoestima, se obtuvieron los siguientes datos (tabla 2):

Tabla 1. Prueba t para muestras independientes

\begin{tabular}{|l|c|c|c|c|}
\hline \multirow{2}{*}{ Variable } & \multicolumn{2}{|c|}{ Media } & \multirow{2}{*}{ t } & $\begin{array}{c}\text { Sig. } \\
\text { bilateral }\end{array}$ \\
\cline { 2 - 3 } & $\begin{array}{c}\text { Tradicional } \\
\mathrm{N}=72\end{array}$ & $\begin{array}{c}\text { Montessori } \\
\mathrm{N}=65\end{array}$ & & .245 \\
\hline Inadaptación & 3.50 & 3.20 & 1.145 & .20 \\
\hline Ansiedad & 49.81 & 47.28 & 1.871 & .064 \\
\hline Autoestima & 46.99 & 48.08 & -.648 & .518 \\
\hline
\end{tabular}

Tabla 2. Correlaciones entre inadaptación, ansiedad y autoestima para la muestra total

\begin{tabular}{|c|c|c|c|}
\hline & & Ansiedad & Autoestima \\
\hline \multirow{3}{*}{ Inadaptación } & Correlación de Pearson & $.563(* *)$ & $-.631(* *)$ \\
\hline & Sig. (bilateral) & .000 & .000 \\
\hline & $\mathrm{N}$ & 137 & 137 \\
\hline \multirow{3}{*}{ Ansiedad } & Correlación de Pearson & & $-.378(* *)$ \\
\hline & Sig. (bilateral) & & .000 \\
\hline & $\mathrm{N}$ & & 137 \\
\hline
\end{tabular}

${ }^{* *}$ La correlación es significativa al nivel 0,01 (bilateral). 
Lo anterior permite aseverar que entre las variables inadaptación y autoestima existe una correlación negativa moderada, significativa al nivel 0.01 bilateral, en tanto que la correlación hallada entre inadaptación y ansiedad es positiva moderada, en un nivel de significancia de 0.01 . Ello indica que a menor grado de autoestima, el nivel de inadaptación se incrementa. En el caso de la asociación entre inadaptación y ansiedad se entiende que a mayor rango de inadaptación, el puntaje de ansiedad se eleva. El análisis entre ansiedad y autoestima muestra una correlación negativa entre débil y moderada, significativa al 0.01 , que permite suponer que a mayor nivel de autoestima, el grado de ansiedad decrece.

Para el análisis factorial de los tres instrumentos aplicados se recurrió al método de extracción de componentes principales y al método de rotación Varimax con Kaiser. Para determinar la pertenencia de los reactivos a determinado factor, se aplicó el criterio de seleccionar reactivos con un peso factorial de .30 o superior, con carga positiva y la mayor, dado el caso de que el reactivo apareciera en dos o más factores. Para establecer la confiabilidad de cada instrumento se analizó el alfa de Cronbach de cada uno.

En referencia al análisis factorial del Tamai, bajo la premisa de que este instrumento tiene como objetivo la autoevaluación de la inadaptación personal, social, escolar y familiar; se exploraron los reactivos que constituían cada uno de estos cuatro factores. La rotación convergió en 9 iteraciones. En observancia a que obtuvieron un peso factorial inferior a .30 , cuatro reactivos fueron eliminados: 1 . "Me gustaría tener menos edad"; 67. "La culpa de lo malo que me pasa la suelen tener los demás"; 70. "Normalmente estoy bien, sin marearme ni ganas de devolver" y 99. "Prefiero ser uno más de la cuadrilla que ser el jefe". Así mismo se indica el número progresivo asignado en el test a cada reactivo (tabla 3).

\section{Descripción de factores:}

Negatividad. Comprende percepciones hostiles del sujeto acerca de sí mismo, del ambiente y de sus padres.

Psicosomático. Incorpora consideraciones del niño sobre sí mismo y sus interacciones con los demás, en algunos casos se hacen referencias explícitas a respuestas físicas y emocionales.

Adaptación escolar y social. Incluye referencias a conductas y actitudes, ya sea de rechazo o aceptación a profesores y a actividades académicas. Del mismo modo se mencionan habilidades sociales.

Afectividad. Conformado por reactivos sobre estilos parentales afectuosos o permisivos.

En cuanto a la Escala de Ansiedad Manifiesta en Niños (revisada) CMAS-R, se refieren cuatro factores y se señala el número adjudicado en el test a cada reactivo (tabla 4). La convergencia de la rotación se dio en 9 iteraciones. El reactivo número 18: "Soy muy senti-

Tabla 3. Análisis factorial del Test autoevaluativo multifactorial de adaptación infantil

\begin{tabular}{|c|c|c|c|c|c|c|}
\hline $\begin{array}{c}\text { Total de } \\
\text { reactivos }\end{array}$ & $\begin{array}{c}\text { Reactivos con } \\
\text { peso factorial } \\
\text { menor a .30 }\end{array}$ & $\begin{array}{c}\text { Factor 1 } \\
\text { Negatividad }\end{array}$ & $\begin{array}{c}\text { Factor 2 Psicoso- } \\
\text { mático }\end{array}$ & $\begin{array}{c}\text { Factor 3 } \\
\text { Inadaptación } \\
\text { escolar y social }\end{array}$ & $\begin{array}{c}\text { Factor } 4 \\
\text { Afectividad }\end{array}$ & $\begin{array}{c}\text { Alfa de } \\
\text { Cronbach de } \\
\text { la Escala Total }\end{array}$ \\
\hline & & & $2-8,10-15,17-20$, & $23-41,54-58$, & & \\
175 & \multirow{2}{*}{$1,67,70,99$} & $9,16,21-22,51$, & $43-50,52-53,60$, & $61,65,78-88$, & 42,113, & $116-124,126-$ \\
& & $59,115,146-$ & $62-64,66,68-69$, & $100-102,104-$ & 11684 \\
& & $147,150-175$ & $71-77,89-98$, & $105,107,111-$ & 145,148 & \\
& & $103,106,108-110$ & $112,114,125$ & & \\
\end{tabular}

Tabla 4. Análisis factorial de la Escala de Ansiedad Manifiesta en Niños (revisada) CMAS-R.

\begin{tabular}{|c|c|c|c|c|c|c|}
\hline $\begin{array}{c}\text { Total de } \\
\text { reactivos }\end{array}$ & $\begin{array}{c}\text { Reactivos con } \\
\text { peso factorial } \\
\text { inferior a .30 }\end{array}$ & $\begin{array}{c}\text { Factor 1 } \\
\text { Inquietud } \\
\mathrm{N}=15\end{array}$ & $\begin{array}{c}\text { Factor 2 } \\
\text { Mentira } \\
\mathrm{N}=8\end{array}$ & $\begin{array}{c}\text { Factor 3 } \\
\text { Preocu- } \\
\text { pación } \\
\mathrm{N}=7\end{array}$ & $\begin{array}{c}\text { Factor 4 } \\
\text { Somático } \\
\mathrm{N}=6\end{array}$ & $\begin{array}{c}\text { Alfa de } \\
\text { Cronbach } \\
\text { de la Escala } \\
\text { Total }\end{array}$ \\
\hline 37 & 18 & $\begin{array}{c}3,5-7,11,13, \\
17,21,23,25, \\
27,29-30,35-36\end{array}$ & $\begin{array}{c}1,4,8,12,16, \\
20,24,28\end{array}$ & $\begin{array}{c}9-10,14-15, \\
22,32,37\end{array}$ & $\begin{array}{c}2,19,26,31, \\
33,34\end{array}$ & .840 \\
\hline
\end{tabular}


mental", alcanzó un peso factorial de .275, por lo que fue eliminado.

Descripción de factores:

Inquietud. Considera reactivos sobre malestares físicos o psicológicos.

Mentira. Agrupa afirmaciones vinculadas a la deseabilidad social.

Preocupación. Conjunta ítems que expresan intranquilidad, nerviosismo.

Somático. Comprende enunciados acerca de reacciones físicas o emocionales negativas.

En el Cuestionario para la evaluación de autoestima en educación primaria (A-EP), se identificaron 3 factores, ningún reactivo fue eliminado, se señala el número asignado en el test a cada uno de los reactivos, al enlistarlos en cada factor (tabla 5). La rotación convergió en 5 iteraciones.

\section{Descripción de factores:}

Competencias escolares. Consiste en ítems que describen habilidades, competencias para el aprendizaje.

Autoestima positiva. Concentra afirmaciones que manifiestan seguridad y confianza, además de respeto hacia sí mismo.

Autoestima negativa. Compuesto de reactivos que expresan autodesprecio y autodes-calificación.

\section{DISCUSIÓN}

El no haber encontrado diferencias estadísticamente significativas entre las medias de los grupos constituidos por alumnos de escuela tradicional y escuela Montessori, tanto en la variable adaptación, como en las variables ansiedad y autoestima puede explicarse en el hecho de que el desarrollo de cada niño se halla determinado no sólo por sus capacidades físicas o mentales, sino por diferentes estructuras (sociedad, escuela, familia, entre otras) y por procesos generados por estas instancias (nivel socioeconómico y cultural, ambiente escolar, relaciones familiares, etc.). La manera en que estos elementos se articulan se rige por un complejo sistema de interacciones que produce efectos únicos en cada individuo. Por tanto, es necesario afirmar que aún y cuando se reconoce el papel fundamental de la escuela en la formación de los individuos, debe tomarse en cuenta la confluencia de factores diversos en su evolución.

Por otra parte, la cercanía de las medias para las tres variables estudiadas hace suponer similar eficacia en el método educativo tradicional y en el Montessori.

Los resultados obtenidos al correlacionar adaptación, ansiedad y autoestima confirman y describen la naturaleza y fuerza de su asociación, lo que permite diseñar estrategias de prevención e intervención tanto a nivel educativo como clínico.

En cuanto a los datos obtenidos a través del análisis factorial, fue posible establecer parámetros de "confiabilidad, validez y estandarización” (Coolican, 2005, p. 128), de los tres instrumentos psicométricos aplicados en esta investigación. En el caso de la confiabilidad, afirma Coolican (2005, p. 129) que un grado aceptable está determinado por un alfa de Cronbach de entre ".75 y hasta 1". A su vez, Aron y Aron (2001, p. 567) establecen que en psicología se espera un alfa de Cronbach "de al menos 0,7 y preferentemente cercana a 0,9 , para que la prueba sea útil”. Tales criterios se cumplen en el alpha de Cronbach de los tres tests analizados: en el caso del "Tamai (Test Autoevaluativo Multifactorial de Adaptación Infantil)”, es de .984; la "Escala de Ansiedad Manifiesta en Niños (revisada) CMAS-R", alcanza .840 y el "Cuestionario para la Evaluación de Autoestima en Educación Primaria (AEP)" obtiene .745 .

Tabla 5. Análisis factorial del Cuestionario para la evaluación de autoestima en educación primaria (A-EP)

\begin{tabular}{|c|c|c|c|c|}
\hline $\begin{array}{c}\text { Total de } \\
\text { reactivos }\end{array}$ & $\begin{array}{c}\text { Factor 1 } \\
\text { Competencias } \\
\text { escolares }\end{array}$ & $\begin{array}{c}\text { Factor 2 } \\
\text { Autoestima } \\
\text { positiva }\end{array}$ & $\begin{array}{c}\text { Factor 3 } \\
\text { Autoestima negativa }\end{array}$ & $\begin{array}{c}\text { Alfa de Cronbach } \\
\text { De la Escala Total }\end{array}$ \\
\hline 17 & $\begin{array}{c}2,5,9,13-14,16-17, \\
\mathrm{~N}=7\end{array}$ & $\begin{array}{c}1,3,6,10-11,15, \\
\mathrm{~N}=6\end{array}$ & $\begin{array}{c}4,7-8,12, \\
\mathrm{~N}=4\end{array}$ & .745 \\
\hline
\end{tabular}


Respecto a los motivos de eliminación de reactivos de las escalas dado su peso factorial inferior a .30, se hace referencia a lo dicho por Coolican (2005, pp. 121-122) sobre la "construcción de afirmaciones", en las que el autor considera inevitables las variaciones en la "interpretación del mundo, incluyendo la interpretación del lenguaje" por parte de los sujetos, ante lo que recomienda "claridad", previniendo "ambigüedades evidentes y factores de predisposición para la mayoría de la población". Como "factores de predisposición" el citado autor enlista: "complejidad", "términos técnicos", "ambigüedad", "reactivos de doble contenido", "negativos", "lenguaje emotivo", "preguntas tendenciosas", "invasión de privacidad", "balance de reactivos escalares" y "sensibillidad de los reactivos de la escala al nivel de medición”. Coolican (2005, pp.122-123) también hace mención dentro del rubro "organización de los reactivos" a posibles causas que llevan al sujeto a distorsionar sus respuestas: el efecto de "propensión de aquiescencia de respuesta", la "interpretación del examinando" y la "deseabilidad social".

Un análisis más profundo de los reactivos eliminados en el análisis factorial del presente estudio identificaría las causas específicas de su baja carga factorial.

\section{CONCLUSIONES}

En el transcurso de la presente investigación se ha constatado que tanto filogenética como ontogenética-

\section{REFERENCIAS}

Agras, S. (1989). Pánico: cómo superar los miedos, las fobias y la ansiedad. Barcelona: Labor.

Aron, A. \& Aron, E. N. (2001). Estadística para psicología. Buenos Aires: Pearson Education.

Bordera Escolano, F. (2005). La intervención en los trastornos traumáticos desde el medio escolar. España: Psicología educativa: Revista de los psicólogos de la educación, 11, 1, 11-26.

Branden, N. (2001). La psicología de la autoestima. México: Paidós.

Carreras de Alba, M. del R., Muñoz, J. M., Braza Lloret, P. \& Braza, F. (2002). Relación entre la flexibilidad comportamental de niños en el periodo de educación mente la adaptación, la ansiedad y la autoestima son mecanismos fundamentales en el desarrollo humano.

Dada la importancia de estos constructos, se considera conveniente recomendar ampliar las líneas de investigación vinculando a su análisis: género, edad, escolaridad, condición socioeconómica, coeficiente de inteligencia, funcionamiento familiar, rasgos de personalidad, rendimiento escolar y otras. Todo ello propiciaría una mejor comprensión tanto de la conducta del sujeto, como de la naturaleza de las variables mencionadas y sus asociaciones.

Así mismo se sugiere profundizar en el estudio de las características, alcances y perspectivas de diferentes métodos educativos, sin dejar de lado el análisis y revisión del modelo tradicional, en consideración a su preeminencia en el mundo entero.

Es indiscutible que para llevar a cabo tales estudios es necesaria la disponibilidad de instrumentos psicométricos idóneos, para lo cual se contemplan dos alternativas: la estandarización de tests extranjeros o la construcción de escalas para muestras nacionales.

Los hallazgos de la presente investigación posibilitan planificar y aplicar estrategias pedagógicas y/o psicológicas pertinentes, a fin de prevenir o solucionar trastornos asociados ya sea a un alto grado de ansiedad o bien, a un bajo nivel de adaptación y/o de autoestima, ello con el fin de optimizar el funcionamiento personal, escolar, social y familiar de los niños.

infantil y su adaptación social posterior. España: Infancia y Aprendizaje, 25, 4, 515-526.

Castellanos, A. G., \& Albizu, C. (2003). A comparison of tradicional vs. Montessori education in relation to children's self-esteem, self-efficacy and prosocial behavior. USA: Dissertation Abstracts International. Section B: The Sciences and Engineering, 63 (11-B), 5548.

Castro Fonseca, A. (1998). Problemas de ansiedade em criancas e adolescentes: elementos para a sua caracterizacao e para o seu diagnostico. Portugal: Revista Portuguesa de Pedagogía, 32, 2, 7-34.

Coolican, H. (2005). Métodos de investigación y estadística en psicología. México: Manual Moderno. 
Darwin, C., (1859/2005). El origen de las especies. México: Grupo Editorial Tomo.

De la Garza, G. (2002). La misión del maestro en el siglo XXI: reflexión sobre la función docente. México: Educación 2001, 83, 25-28.

Di Giorgi, P. (1977). El niño y sus instituciones: la familia, la escuela. México: Roca Pedagogía.

Echeburúa Odriozola, E. (1998). Trastornos de ansiedad en la infancia. Madrid: Pirámide.

Gallardo Cruz, J. A. \& Jiménez Hernández, M. (1997). Efectos del maltrato y del status sociométrico sobre la adaptación social y afectivo infantil. España: Psicothema, 9, 1, 119-131.

González Martínez, M. T., y García González, M. L. A. (1995). El estrés y el niño: factores de estrés durante la infancia. España: Aula. Revista de enseñanza e investigación educativa, 7, 185-201.

Gray, J. (1971). The psychology of Fear and Stress. New York: McGraw-Hill.

Gutiérrez Navarrete, J. J., Lerma Mayer, A. I. \& Schroeder Vásquez, C. (2009). Socialización en niños de 11-12 años en escuelas tradicional y Montessori. México: Odiseo. Revista Electrónica de Pedagogía. Recuperado el 20 de agosto de 2009, de http.//www.odiseo.com. $\mathrm{mx} /$ bitácora-educativa/socializacion-ninyos-1112años-escuela-tradicional-montessori.

Hernández Hernández, P. (2004). Tamai: Test autoevaluativo multifactorial de adaptación infantil. Madrid: Tea Ediciones.

Hess, R. D. \& Croft, D. J. (1981). Libro para educadores de niños en edad preescolar: texto básico. México: Diana.
Jaik Dipp, A., Serrano Morales, J. A., López González, C. \& Amancio Rosas G. (2008). Estudio comparativo entre dos modelos pedagógicos a nivel preescolar. México: Investigación Educativa Duranguense, 9, julio, 21-32.

López, F. \& Cantero López, M. J. (2004). Período de adaptación escolar: descripción del proceso y su supuesta universalidad cuando los menores ingresan a los 3 años. España: Infancia y Aprendizaje, 27, 1, 27-41.

Merani, A. L. (1978). Psicología genética. Barcelona: Grijalbo.

Mishra, R. C., (1988). Learning strategies among children in the modern and traditional schools. India: Indian Psychologist, 5, 1, 17-24.

Montt s., M. E. \& Ulloa Ch., F. (1996). Autoestima y salud mental en adolescentes. Chile: Salud Mental, 19, 3, 30-35.

Palacios, J., Marchesi, A. \& Coll, C., (Comps.) (2002). Desarrollo psicológico y educación: 1. Psicología evolutiva. Madrid: Alianza editorial.

Pruneda Huidobro, E. (2003). “QQué es la autoestima?”. México: Rompan filas, 12, 63, 10-15.

Ramos Álvarez, R., Jiménez López, A. I., Muñoz-Addell, Ma. A. \& Lapaz Cruz, E. (2006). Cuestionario para la evaluación de autoestima en educación primaria. Madrid: Tea Ediciones.

Reynolds, C. R. \& Richmond, B. O. (1985). Escala de Ansiedad Manifiesta en Niños (revisada) CMAS-R. (1985). México: El Manual Moderno.

Schaffer, R. (2000). Desarrollo social. México, Siglo XXI. 